\title{
The clinical significance of IL-6s and IL-27s in Bronchoalveolar lavage fluids from children with mycoplasma pneumoniae pneumonia
}

Jie Zhao

Zibo Central Hospital

Yuyun Li

Zibo Central Hospital

Wen Zhang ( $\sim$ zangyua@yeah.net)

Zibo Central Hospital

\section{Research article}

Keywords: mycoplasma pneumoniae pneumonia, Bronchoalveolar lavage fluids, IL-6; IL-27, communityacquired pneumonia

Posted Date: April 13th, 2020

DOI: https://doi.org/10.21203/rs.2.15861/v4

License: (c) (i) This work is licensed under a Creative Commons Attribution 4.0 International License.

Read Full License

Version of Record: A version of this preprint was published at BMC Infectious Diseases on May 11th, 2020. See the published version at https://doi.org/10.1186/s12879-020-05017-3. 


\section{Abstract}

Background: IL-6 was associated with the severity of mycoplasma pneumoniae pneumonia (MPP). But the relationship between IL-27 and MPP was unknown.

Methods: 98 patients with MPP < 14 years old were enrolled in this study and divided into groups by severity (mild cases and severe cases), infection types (MP single infection group and MP mixed infection group) and DNA loads (low MP DNA loads group and high MP DNA loads group), respectively. 15 children with foreign bodies in bronchus were also enrolled as control. IL-6s and IL-27s in bronchoalveolar lavage fluids (BALFs) from these children were measured by ELISA.

Results: There were significant differences in IL-6s of BALFs from patients between mild cases and severe cases, MP single infection group and MP mixed infection group, and low MP DNA loads group and high MP DNA loads group, respectively (Pख0.05). Compared with IL-6s of BALFs from control, IL-6s in BALFs from the 6 patient groups were significantly higher (P®0.05). IL-27s in BALFs from MP mixed infection group were significantly lower than those from MP single infection group and control (Pख0.05) respectively.

Conclusion: IL-6 was firmly associated with MPP and had potential application in clinical practice while IL-27 was not related to MP infection.

\section{Background}

Mycoplasma pneumoniae (MP) is one of the main pathogens in respiratory infections in children. It causes more than $40 \%$ of community-acquired pneumonia (CAP) cases in children, of which $18 \%$ cases need hospitalization [1]. At present there are many problems with patients suffering from Mycoplasma pneumoniae pneumonia (MPP) such as the increasing macrolide resistance rate ${ }^{[2]}$, the complex multiple systemic complications ${ }^{[3]}$, the increasing occurrence of refractory MPP ${ }^{[4]}$. Therefore, MPP has attracted great attentions from many practitioners and patients. Immune function disorders are involved in the pathogenesis of MPP ${ }^{[5]}$. And IL-6 plays important role in regulating immune functions ${ }^{[6]}$. It is involved in the infection process of MP and plays an important role in the pathogenesis of MPP [7]. One study suggests that IL-6 is associated with the severity of MPP [8]. IL-27 is another important cytokine which is firmly associated with IL-6. It can induce the secretion of IL-6 ${ }^{[9]}$ and can also block the activity of IL-6 by its subunit of IL-27 p28 [10] which plays dual roles of pro-inflammation and anti-inflammation. Plfans et $\mathrm{al}^{[11]}$ reports that IL27 is positively associated with IL 6 in patients with brain injury. However, no reports have been found about whether there is any relationship between them in MPP.

Fiberoptic bronchoscopy and bronchoalveolar lavage is safe and effective in the diagnosis and treatment of MPP, which can provide bronchoalveolar lavage fluids (BALFs) for research. BALFs can reflect the pathological and biochemical changes of lung tissues directly. However, there have been few reports 
about IL-6s and IL-27s in BALFs from MPP patients. Only a few reports about the relationship between IL6 in sera and the severity of MPP ${ }^{[8]}$ can be found.

In this study, the levels of IL-6s and IL-27s in BALFs from MPP patients and control were measured to explore their clinical significances.

\section{Methods}

\subsection{Including, excluding, and grouping criteria}

In this study, the diagnosis of MPP met the following criteria: 1) fever, coughing, and other respiratory tract infection symptoms; 2) chest radiographic examinations with bronchial pneumonia, interstitial pneumonia, segmental or lobar pneumonia, and even pleural effusion; and 3) a single serum anti- MP IgM antibody titer of $\geq 1: 160$ at the acute phase following admission (in those with no history of respiratory infections in the past 3 months) and a positive PCR test for MP. Patients with any of the following criteria [12-13] would be diagnosed as severe cases: 1 ) tachypnea or tachycardia (Tachypnea was defined as a respiratory rate of $>40 / \mathrm{m}$ for children aged $1-5$ years, and $30 / \mathrm{m}$ for children aged $>5$ years. Tachycardia was defined as $>140 \mathrm{bpm}$ for children aged $1-3$ years, $>120 \mathrm{bpm}$ for children aged $3-5,>118 \mathrm{bpm}$ for children aged 5-10 years, and $>100 \mathrm{bpm}$ for children aged $>10$ years of age.) with or without nasal flare, moaning, three concave sign, and cyanosis; 2 ) hypoxemia (SaO2 $\leq 92 \%)$; 3 ) refractory MPP; 4) multilobar involvement or involvement area $\geq 2 / 3$ on chest radiographs; 5) pleural effusion (>300ml), severe atelectasis, pulmonary necrosis and pulmonary abscess. 6) Other severe complications (central nervous system infections, heart failure, myocarditis, gastrointestinal hemorrhage, and obvious electrolyte/ acidbase balance disorders). Patients having infections within 3 months, or suffering from known coexisting chronic, progressive or oncological illnesses, or receiving corticosteroids or immunosuppressive agents within 3 months, or with immune hypofunctions or immune related diseases, or allergic diseases or suspected allergic diseases (including allergic rhinitis and atopic dermatitis) or asthmas were excluded from the research.

All the patients were divided into severe cases and mild cases by the severity of the diseases. The whole patients could also be divided into MP single infection group and MP mixed infection group according to

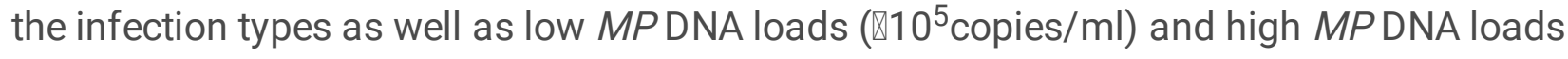
( $\geq 10^{5} \mathrm{copies} / \mathrm{ml}$ ) according to the MP DNA loads.

\subsection{Data collections}

Data including ages, genders, clinical signs and symptoms, laboratory and radiological findings were collected from patients during Jan $1^{\text {st }}$ and Dec $31^{\text {st }}$ of the year 2017. All chest radiographs and computed tomography were reviewed by two experienced radiologists and they agreed on the conclusions.

1.3 MP DNA extractions, detections and quantifications 
MP DNAs from BALFs were extracted using QIAamp DNA MINI kit (Qiagen, Hilden Germany). The target gene for detecting MP by PCR was a segment of gene p1 adhesion with 150 bp (P1-178:

CAATGCCATCAACCCGCGCTTAACC,P1-331: CGTGGTTTGTTGACTGCCACTGCCG). The PCR conditions were: 30 cycles of $94^{\circ} \mathrm{C}$ for $30 \mathrm{~s}, 62^{\circ} \mathrm{C}$ for $30 \mathrm{~s}$ and $72^{\circ} \mathrm{C}$ for $30 \mathrm{~s}$. MP DNA was quantified using Mycoplasma pneumoniae DNA Fluorescence Diagnostic Kit (Shengxiang Biotechnology Co. Ltd, Hunan Province, China) with ABI PRISM 7500 instrument (Applied Biosystems TM, Foster City, California, United States). The experiments were strictly conducted in accordance with the manufacturers' instructions.

\subsection{Collections of BALFs}

Bronchoscopy was performed within 3 days after hospital admission for patients with MPP and immediately after hospital admission to remove the foreign bodies in bronchus from children in control. Flexible fiber optic bronchoscopy and bronchoalveolar lavage were performed following the guidelines described previously ${ }^{[14]}$. BALFs were collected from these children and stored in $-80{ }^{\circ} \mathrm{C}$ freezer.

\subsection{The assays of IL-6s and IL-27s}

IL-6s and IL-27s in BALFs from the children were measured by sandwich enzyme-linked immunosorbent assay (ELISA) with commercial reagent kits (Abcan Company, USA). The experiments were strictly conducted in accordance with the manufacturer's instructions.

\subsection{Statistical analysis}

Statistical analyses were performed using SPSS21.0 Statistical package. Continuous variables were reported as means \pm standard deviations. ANOVA was used to compare means of multiple groups. LSD test was used for the inter-comparison of 2 means in the multiple groups. The ages and genders between MPP patients and control were compared with $t$ test and $x^{2}$ test, respectively. MP DNA loads, IL-6s and IL$27 s$ were shown and compared by box plots. $P \otimes 0.05$ was considered to indicate a statistically significant difference.

\section{Results}

\subsection{General information}

From Jan $1^{\text {st }}$ to Dec $31^{\text {st }}$ of the year 2017, a total of 98 hospitalized children with MPP according to the including criteria and excluding criteria were enrolled. All patients including 47 male patients and 51 female ones underwent fiberoptic bronchoscopy, and BALFs were collected from them. The ages of the patients ranged from 1 to 13 years $(6.63 \pm 2.56$ years). During the study period, 15 children with foreign bodies in bronchus consisting of 8 males and 7 females were also enrolled as control. The ages of them were among 1 to 9 years ( $5.40 \pm 2.27$ years). There were no inflammatory changes on chest radiographs and computed tomography of children in control. No symptoms of respiratory infections including coughing, fever and sore throat existed after brochoscopy in control. WBCs and CRPs in sera of the 
control were in normal ranges. No significant differences in ages $(t=1.76, p \otimes 0.05)$ and sex ratio $\left(x^{2}=0.15, p\right.$ $\otimes 0.05$ ) between the patients and control were observed.

Severe cases consisted of 26 male patients and 27 female ones. The ages of them were $6.91 \pm 2.22$ years old. Mild cases included 45 patients with 21 boys and 24 girls. The ages of them averaged $6.05 \pm 2.73$ years old. MP single infections (60 cases) consisted of 25 male patients and 35 female ones. The ages of them were $6.81 \pm 2.67$ years old. MP mixed infections included 38 patients with 22 boys and 16 girls. The

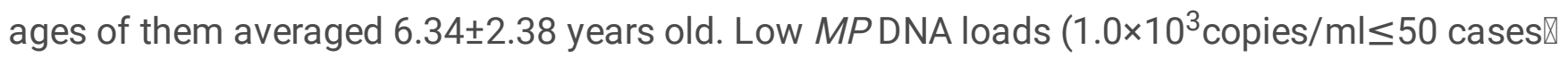

$10^{5}$ copies $/ \mathrm{ml}$ ) consisted of 23 male patients and 27 female ones. The ages of them were $6.92 \pm 2.86$ years old. High MP DNA loads ( 48 cases $\geq 10^{5}$ copies $/ \mathrm{ml}$ ) included 48 patients with 24 boys and 24 girls. The ages of them averaged $5.86 \pm 2.67$ years old. No significant differences in ages $(t=1.72, p \rrbracket 0.05 ; t=0.88, p \rrbracket$ $0.05 ; \mathrm{t}=1.89, \mathrm{p} \otimes 0.05)$ and sex ratio $\left(\mathrm{x}^{2}=0.06, \mathrm{p} \otimes 0.05 ; \mathrm{x}^{2}=2.45, \mathrm{p} \otimes 0.05 ; \mathrm{x}^{2}=0.16, \mathrm{p} \otimes 0.05\right)$ were observed between severe cases and mild cases, MP single infections and MP mixed infections as well as low MP DNA loads and high MPDNA loads.

2.2 MP DNA loads

After confirmation by PCR, all the samples from BALFs were quantified by quantitive real-time PCR with MP DNA loads ranging from $2.98 \times 10^{3}$ copies $/ \mathrm{ml}$ to $1.47 \times 10^{9}$ copies $/ \mathrm{ml}$. (Fig 1, Fig2)

2.2 The comparison of IL-6s and IL-27s in BALFs among severe cases, mild cases and control, respectively

IL-6s in BALFs from severe cases were higher than those from control and mild cases. There were significant differences between them $(p<0.05)$. IL-6s in BALFs from mild cases were significantly higher than those from control $(p<0.05)$ (Fig3 A). It suggested that IL-6s in BALFs were firmly associated with the severity of the disease. By ROC curve analysis (Fig4), the cut off value of IL-6 was $63.055 \mathrm{pg} / \mathrm{L}$. The sensitivity and specificity were $98.10 \%$ and $85.00 \%$, respectively. IL-27s in BALFs from severe cases and mild cases were slightly decreased than those from control, but there were no significant differences in IL27s among them ( $p>0.05)$. (Table 1)(Fig3 D)

2.3 The comparison of IL-6s and IL-27s in BALFs among MP single infection group, MP mixed infection group and control, respectively

IL-6s in BALFs from MP mixed infection group were higher than those from control and MP single infection group. There were significant differences between them $(\mathrm{p}<0.05)$. IL-6s in BALFs from MP single infection group were significantly higher than those from control $(p<0.05)$ (Fig3 B). It suggested that IL-6s in BALFs were associated with MP mixed infections. IL-27s in BALFs from MP mixed infection group were significantly lower than those from control and $M P$ single infection group $(p<0.05)$, which suggested that IL-27 was negatively associated with other pathogens but MP. But no significant differences were found in IL-27s from BALFs between MP single infection group and control ( $p>0.05)$. (Table 2) (Fig3 E) 
2.4 The comparison of IL-6s and IL-27s in BALFs among high MP DNA loads group, low MP DNA loads group and control, respectively

IL-6s in BALFs from high MP DNA loads group were higher than those from control and low MPDNA loads group. There were significant differences between them $(p<0.05)$ (Fig3 C). The levels of IL-6 in BALFs from low MP DNA loads group were also significantly higher than those from control $(p<0.05)$. There were no significant differences in IL-27s in BALFs from high MP DNA loads group, Iow MPDNA loads group and control ( $p>0.05$ ). (Table 3) (Fig3 F)

\section{Discussion}

MPP is a common respiratory infection in children and a leading cause of death in China ${ }^{[15]}$. In recent years, patients infected by MP have increased year by year in the worldwide ${ }^{[16]}$. So MPP has drawn much attention from medical practitioners. The main clinical manifestations include fever, coughing, shortness of breath and continuous dry and wet rales in the lung. Patients often present from mild to severe symptoms. It is reported that IL-6 is associated with the severity of MPP ${ }^{[8]}$. But no reports about IL-6 relating to MP DNA loads and MP infection types in patients with MPP have been found. There are also no reports found about the relationship between IL-27 and MPP. So the research aimed to explore the relationship of IL-6 and MPP further as well as IL-27 and MPP.

IL-6 is an important cytokine that has dual functions in the process of inflammation ${ }^{[6]}$. It is mainly secreted by Th2 cells and can promote the secretion of protective antibodies to extracellular microbial pathogens ${ }^{[6]}$. IL-6 plays an important role in the pathogenesis of MPP ${ }^{[7]}$. Our previous research suggested that IL-6-174 G/C genotype increased MP infection in patients ${ }^{[17]}$, which also suggested that IL-6 was closely related to MP infection. In the research, IL-6s in BALFs were significantly higher in patients with MPP than those in control. There were significant differences in IL-6s between severe cases and mild cases. It suggested that IL- 6 was closely related to the severity of the disease, which was similar to the previous report ${ }^{[8]}$. IL-6s in MP mixed infection group were significantly higher than those in MP single infection group, which suggested that IL-6 may be associated with other pathogens ${ }^{[18]}$. IL-6 was closely associated with MPDNA loads for there were significant differences in IL-6s of BALFs between high MPDNA loads group and low MPDNA loads group. These results had not been found reported previously. Additionally, IL-6s in RMPP patients were significant higher than those in General MPP patients [19]. IL-6 was also associated with pleural effusion ${ }^{[20]}$ and radiological appearance ${ }^{[8]}$ in MPP patients. Guo $L$ et al reported that IL-6s in serum of patients infected by macrolide-resistant strains were higher than those infected by non macrolide-resistant strains ${ }^{[21]}$. Therefore IL- 6 was closely related to MPP. In the future, it may be used as an indicator for reflecting the severity and the infection state of the disease.

IL-27 is produced by antigen-presenting cells upon exposure to microbial-derived molecules and inflammatory stimuli ${ }^{[22]}$. It has emerged as a pro-inflammatory factor that signals via binding to IL-27R, which consists of IL-27Ra (WSX-1/TCCR) and glycoprotein 130 subunits, and mediates various inflammation-promoting biological activities involved in the pathogenesis of many inflammation-related 
diseases ${ }^{[23]}$. Increasing evidence suggests that IL-27 is a strong inducer of chemokines and proinflammatory cytokines including IL- 6 by activated neutrophils, monocytes, and macrophages ${ }^{[9]}$. IL-27 is a heterodimeric cytokine constituted of two subunits, EBI3 and IL-27-p28 ${ }^{[11]}$, and IL-27 p28 can block the activity of IL-6 ${ }^{[10]}$. Therefore, IL-27 may maintain the level of IL-6 in a balance state.

In this research, there were no significant differences in the levels of IL-27 between MPP groups and control, MP severe cases and MP mild cases, high MP DNA loads and low MP DNA loads, which suggested that IL-27 was not related to MPinfection. However, the levels of IL-27 in MP mixed infection group were significantly lower than those in MP single infection group and control, which suggested that IL-27 may be negatively related with other pathogens. Further research could be conducted with the increasing pneumonias infected by other pathogens in our hospital. However, it was postulated that IL-27 was not involved in the pathogenesis of MPP.

The research still had some limitations. The samples size included in the study was not large enough. The pathogens in the MP mixed infection group included many kinds of pathogens such as respiratory syncytial virus, chlamydia pneumonia, and influenza $A$ virus. The number of each kind of pathogens coinfected in the MP mixed infection group was too few to get a statistical analysis. Therefore, the association of IL-6, IL-27 and other pathogens was hard to analyze.

\section{Conclusion}

In summary, IL-6 was closely related with the severity, MPDNA loads, and MP mixed infections in the patients with MPP. It was a potential indicator in clinical practice. However, IL-27 was not related to MP infections but may be related to other pathogens. Both of them should be studied further.

\section{Abbreviations}

MPP mycoplasma pneumoniae pneumonia

MP mycoplasma pneumoniae

BALFs bronchoalveolar lavage fluids

CAP community-acquired pneumonia

\section{Declarations}

Competing interests

The authors declare no competing interests.

Acknowledgements 
We thank the hospital pediatricians and clinical teams on all the pediatric wards who provide care to the children.

Funding

This research did not receive any specific grant from funding agencies in the public, commercial, or notfor-profit sectors.

Availability of Data and Materials

The datasets used and/or analyzed during the current study are available from the corresponding author on reasonable request.

Authors' contributions

LYY and ZJ conceptualized the study. LYY and ZJ were responsible for data curation, formal analysis, doing the experiment and writting the original draft. ZW was responsible for resources, supervision, validation, visualization and experiments. All authors read and approved the final manuscript.

Ethics approval and consent to participate

The study followed the principles of the Declaration of Helsinki and was approved by the Ethics Committee of Zibo Central Hospital. Written informed consents were obtained from the guardians of the patients.

Consent for publication

All authors have read and approved the manuscript for publication.

\section{References}

1. Sondergaard MJ, Friis MB, Hansen DS, Jorgensen IM. Clinical manifestations in infants and children with Mycoplasma pneumoniae infection. PLoS One. 2018;13: e0195288.

2. Cheong KN, Chiu SS, Chan BW, To KK, Chan EL, Ho PL. Severe macrolide-resistant Mycoplasma pneumoniae pneumonia associated with macrolide failure. J Microbiol Immunol Infect. 2016; 49:127-130.

3. Chaudhry R, Ghosh A, Chandolia A. Pathogenesis of Mycoplasma pneumoniae: An update. Indian J Med Microbiol. 2016; 34: 7-16.

4. Inamura N, Miyashita N, Hasegawa S, Kato A, Fukuda Y, Saitoh A, et al. Management of refractory Mycoplasma pneumoniae pneumonia: Utility of measuring serum lactate dehydrogenase level. $J$ of Infect Chemother. 2014; 20: 270-273.

5. 5. Saraya T. Mycoplasma pneumoniae infection: Basics. J Gen Fam Med. 2017; 18: 118-125. 
6. Akdis M, Aab A, Altunbulakli C, Azkur K, Costa RA, Crameri R, et al. Interleukins (from IL-1 to IL-38), interferons, transforming growth factor beta, and TNF-alpha: Receptors, functions, and roles in diseases. J Allergy Clin Immuno.I 2016; 138: 984-1010.

7. He J, Liu M, Ye Z, Tan T, Liu X, You X, et al. Insights into the pathogenesis of Mycoplasma pneumoniae. Mol Med Rep. 2016; 14: 4030-4036.

8. Hsieh CC, Tang RB, Tsai CH, Chen W. Serum interleukin-6 and tumor necrosis factor-alpha concentrations in children with mycoplasma pneumonia. J Microbiol Immunol Infect. 2001; 34: 109112.

9. Guzzo C, Che Mat NF, Gee K. Interleukin-27 induces a STAT1/3- and NF-kappaB-dependent proinflammatory cytokine profile in human monocytes. J Biol Chem. 2010; 285: 24404-24411.

10. Stumhofer JS, Tait ED, Quinn WJ $3^{\text {rd }}$, Hosken N, Spudy B, Goenka R, et al. A role for IL-27p28 as an antagonist of gp130-mediated signaling. Nat Immunol. 2010; 11: 1119-1126.

11. Pflanz S, Timans JC, Cheung J, Rosales R, Kanzler H, Gilbert J, et al. IL-27, a heterodimeric cytokine composed of EBI3 and p28 protein, induces proliferation of naive CD4+ T cells. Immunity. 2002; 16: 779-790.

12. Jiang ZF, Shen KL, Shen Y, Ni X, Yu G, Ma L, et al. Zhu Futang Practice of Pediatrics $8^{\text {th }}$ Edition. People's Medical Publishing House of China. 2015; P:1253-1282.

13. Cho YJ, Han MS, Kim WS, Choi EH, Choi YH, Yun KW, et al. Correlation between chest radiographic findings and clinical features in hospitalized children with Mycoplasma pneumoniae pneumonia. PLoS One. 2019; 14:e0219463.

14. Wang K, Gao M, Meng F, Li D, Lu R, Wang Y, et al. Transcriptome analysis of bronckoalveolar lavage fluid from children with severe mycoplasma pneumoniae pneumonia reveals novel gene expression and immunodeficiency. Hum Genomics. 2017;11(1):4-4.

15. Tashiro M, Fushimi K, Kawano K, Takazono T, Saijo T, Yamamoto K, et al. Adjunctive corticosteroid therapy for inpatients with Mycoplasma pneumoniae pneumonia. BMC Pulm Med . 2017;17: 219.

16. Qiu L, Wang L, Tan L, Li M, Wu C, Li L, et al. Molecular characterization of genomic DNA in Mycoplasma pneumoniae strains isolated from serious mycoplasma pneumonia cases in 2016, Yunnan, China. Infect Genet Evol. 2018; 58: 125-134.

17. Zhao J, Zhang W, Shen L, Yang X, Liu Y, Gai Z. Association of the ACE, GSTM1, IL-6, NOS3 and CYP1A1 polymorphisms with susceptibility of mycoplasma pneumoniae pneumonia in Chinese Children. Medicine (Baltimore). 2017; 96: e6642 .

18. Dienz O, Rud JG, Eaton SM, Lanthier PA, Burg E, Drew A, et al. Essential role of IL-6 in protection against H1N1 influenza virus by promoting neutrophil survival in the lung. Mucosal Immunol . 2012; 5: 258-266.

19. Zhang Y, Mei S, Zhou Y, Huang M, Dong G, Chen Z. Cytokines as the good predictors of refractory Mycoplasma pneumoniae pneumonia in school-aged children. Sci Rep. 2016; 6: 37037 .

20. Zhao JL, Wang X, Wang YS. Relationships between Th1/Th2 cytokine profiles and chest radiographic manifestations in childhood Mycoplasma pneumoniae pneumonia. Ther Clin Risk Manag. 2016; 12: 
1683-1692.

21. Guo L, Liu F, Lu MP, Zheng Q, Chen ZM. Increased T cell activation in BALF from children with Mycoplasma pneumoniae pneumonia. Pediatr Pulmonol. 2015; 50: 814819.

22. Villarino AV, Larkin J 3rd, Saris CJ, Caton AJ, Lucas S, Wong T, et al. Positive and negative regulation of the IL-27 receptor during lymphoid cell activation. J Immunol. 2005; 174: 7684-7691.

23. Neurath MF. New therapies for sepsis: focus on the interleukin (IL) 12 family member IL27. Ann Rheum Dis Suppl. 2007; 3: iii29-iii31.

\section{Tables}

Table1. The comparison of IL-6s and IL-27s in BALFs among three groups of children respectively

\begin{tabular}{llll}
\hline Groups & cases & IL-6s $(\mathrm{pg} / \mathrm{ml})$ & IL-27s $(\mathrm{pg} / \mathrm{ml})$ \\
\hline MPP severe cases & 53 & $98.13 \pm 21.01^{\dagger}$ & $151.40 \pm 26.77$ \\
MPP mild cases & 45 & $46.62 \pm 26.68^{\dagger}$ & $144.75 \pm 18.77$ \\
control & 15 & $10.06 \pm 2.26$ & $161.62 \pm 13.35$ \\
F value & & 119.98 & 2.74 \\
P & & 0.00 & 0.07
\end{tabular}

Note: “†” signified that there was significant difference between MPP cases and control. “" signified that there was significant difference between MPP severe cases and MPP mild cases.

Table2. The comparison of IL-6s and IL-27s in BALFs among three groups of children respectively

\begin{tabular}{llll}
\hline Groups & cases & IL-6s $(\mathrm{pg} / \mathrm{ml})$ & IL-27s $(\mathrm{pg} / \mathrm{ml})$ \\
\hline MP single infection & 60 & $62.82 \pm 28.52^{\dagger}$ & $166.77 \pm 13.23$ \\
MP mixed infection & 38 & $92.89 \pm 36.70^{\dagger}$ & $119.26 \pm 9.37 \dagger$ \\
control & 15 & $10.06 \pm 2.26$ & $161.62 \pm 13.35$ \\
F value & & 42.14 & 187.57 \\
P & & 0.00 & 0.00 \\
\hline
\end{tabular}

Note " + " signified that there was significant difference between MPP cases and control. “】” signified that there was significant difference between MPP mixed infection group and MPP single infection group. 
Table3. The comparison of IL-6s and IL-27s in BALFs among three groups of children respectively

\begin{tabular}{llll}
\hline groups & cases & IL-6s $(\mathrm{pg} / \mathrm{ml})$ & IL-27s $(\mathrm{pg} / \mathrm{ml})$ \\
\hline high MP DNA loads & 50 & $101.71 \pm 11.311^{-\square}$ & $144.90 \pm 27.74$ \\
low MP DNA loads & 48 & $41.26 \pm 5.47 \dagger$ & $151.93 \pm 24.04$ \\
control & 15 & $10.06 \pm 2.26$ & $161.62 \pm 13.35$ \\
F value & & 87.48 & 2.86 \\
P & & 0.00 & 0.06 \\
\hline
\end{tabular}

Note “†” signified that there was significant difference between MP DNA loads and control. "[" signified that there was significant difference between high MP DNA loads group and low MP DNA loads group.

\section{Figures}

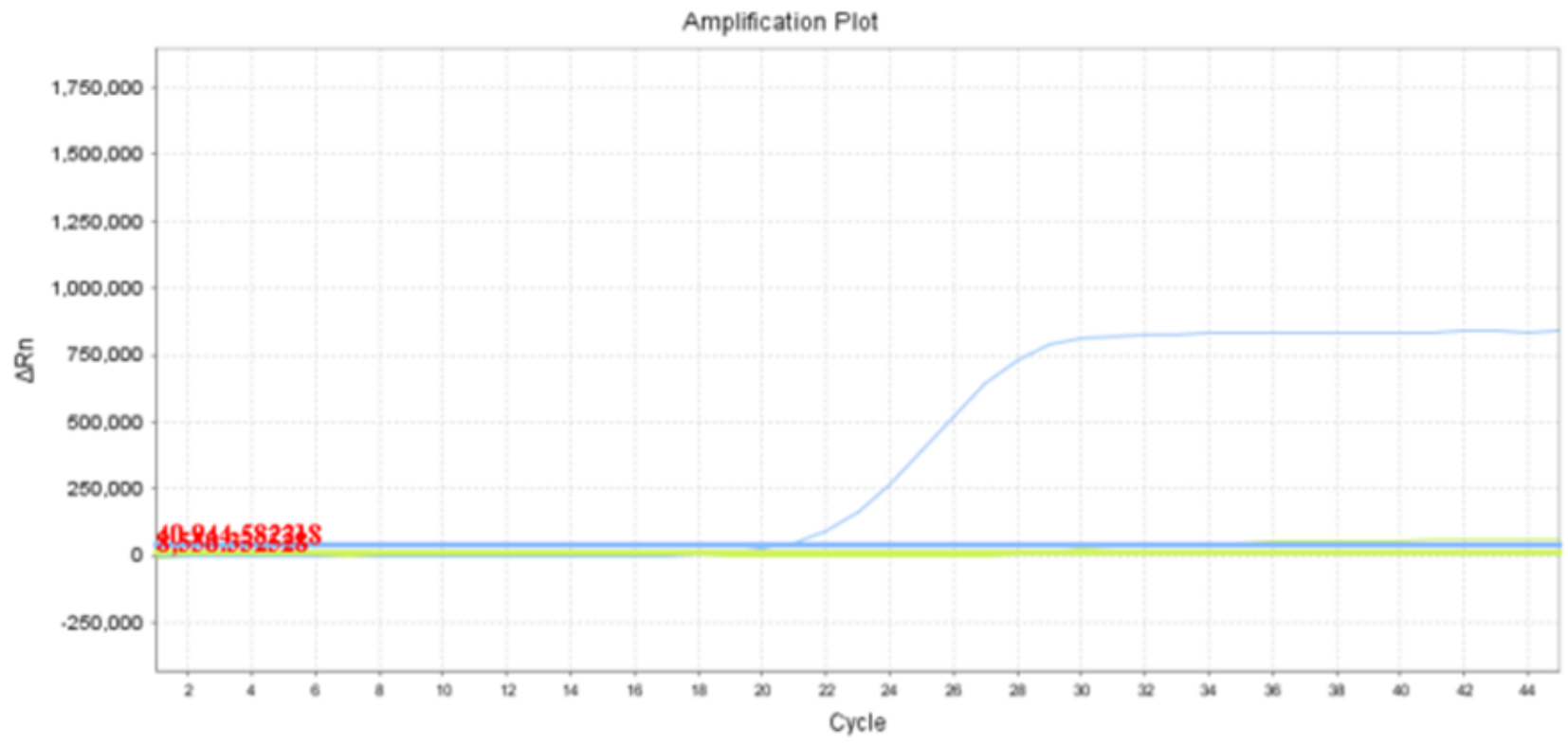

\section{Figure 1}

The amplification plot of one sample $(2.9 * 107$ copies $/ \mathrm{ml})$ 


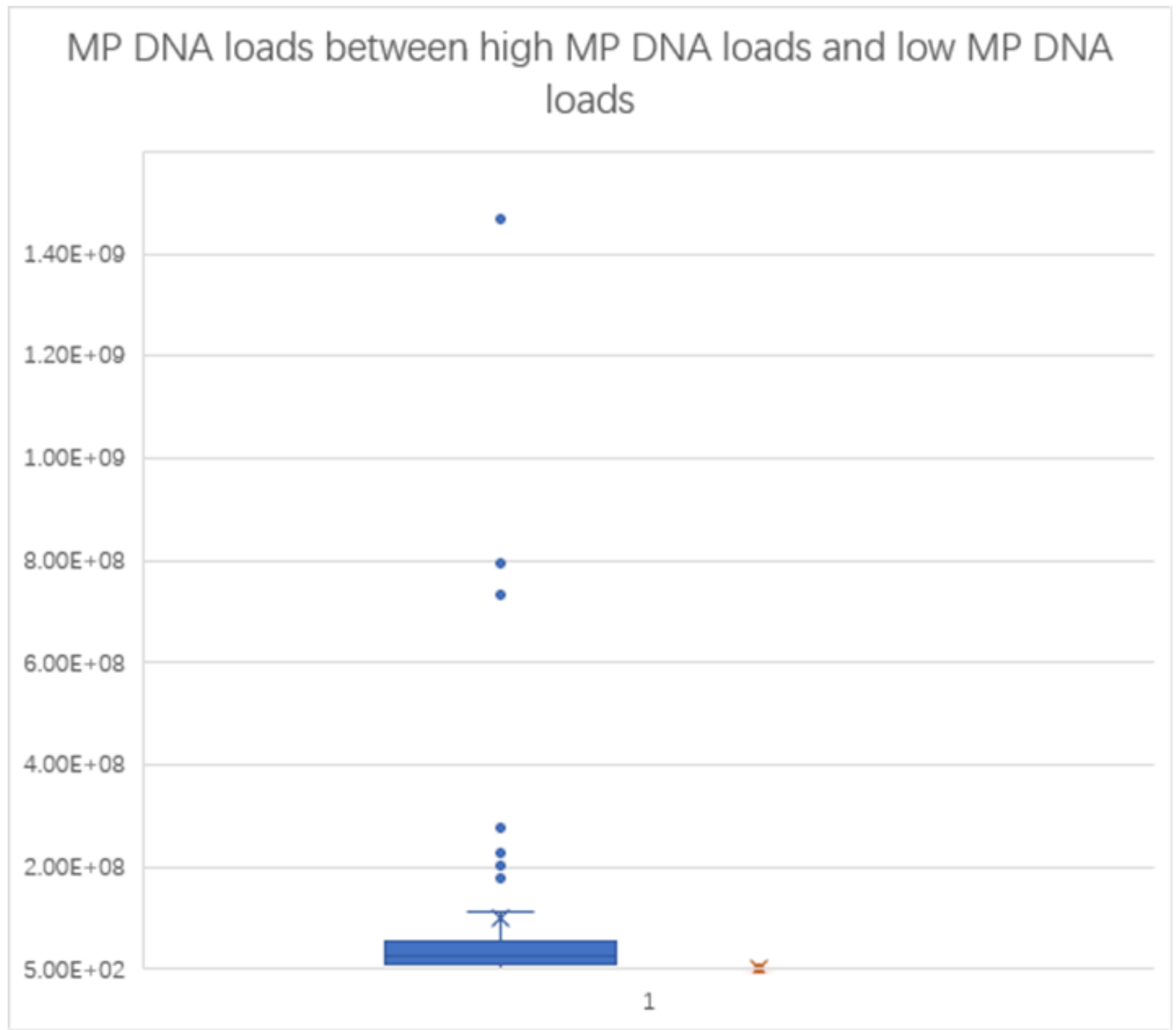

Figure 2

Box plot of MP DNA loads 
The levels of IL-6 in severe cases, mild cases and control, repectively

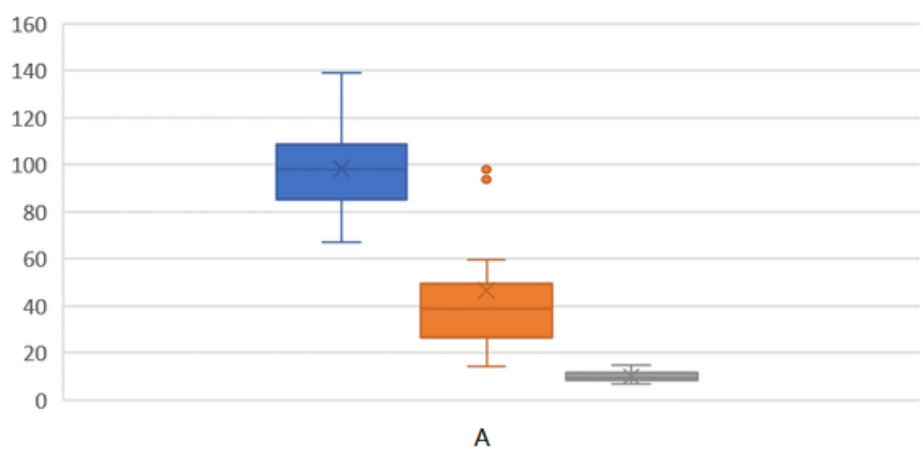

The levels of IL-6 in high MP DNA loads group, low MP DNA loads group and control, respectively

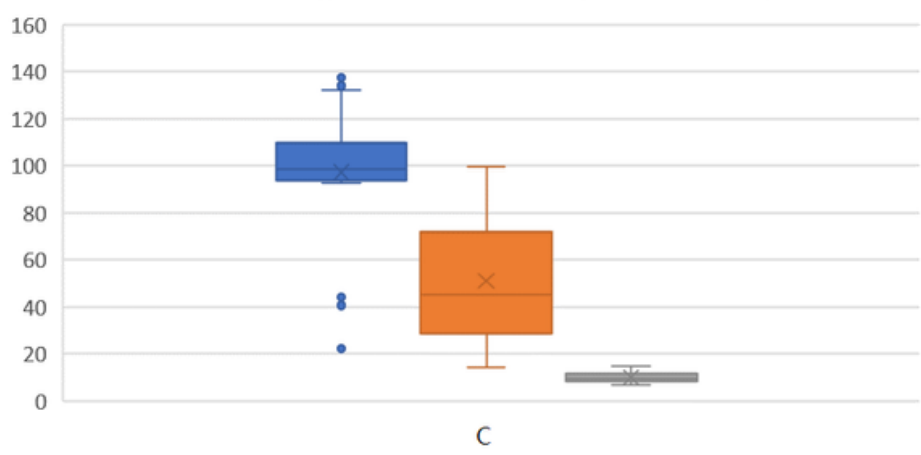

The levels of IL-27 in MP single infection group, MP mixed infection group and control, respectively

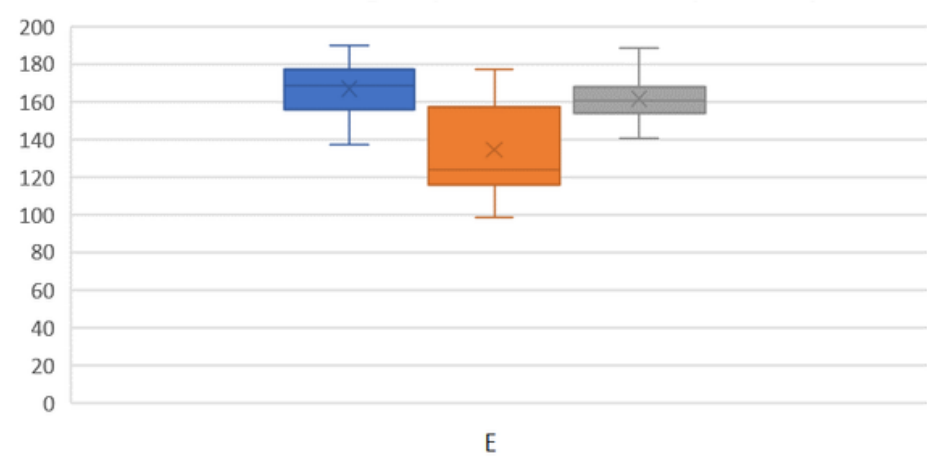

The levels of IL-6 in MP single infection group, MP mixed infection group and control, respectively

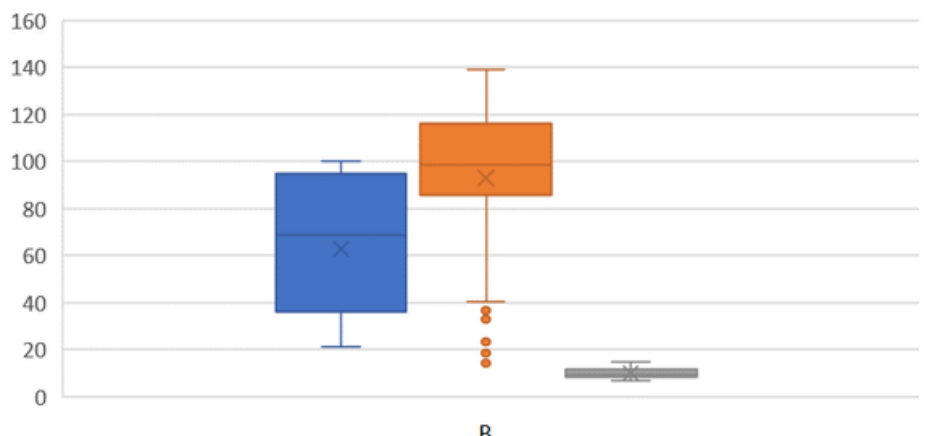

The levels of IL-27 in sever cases, mild cases and control, respectively

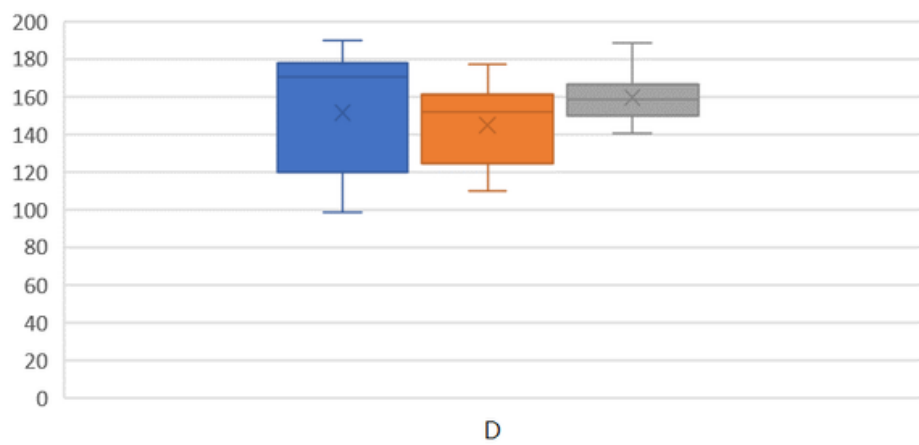

The levels of IL-27 in high MP DNA loads group, low MP DNA loads group and control, respectively

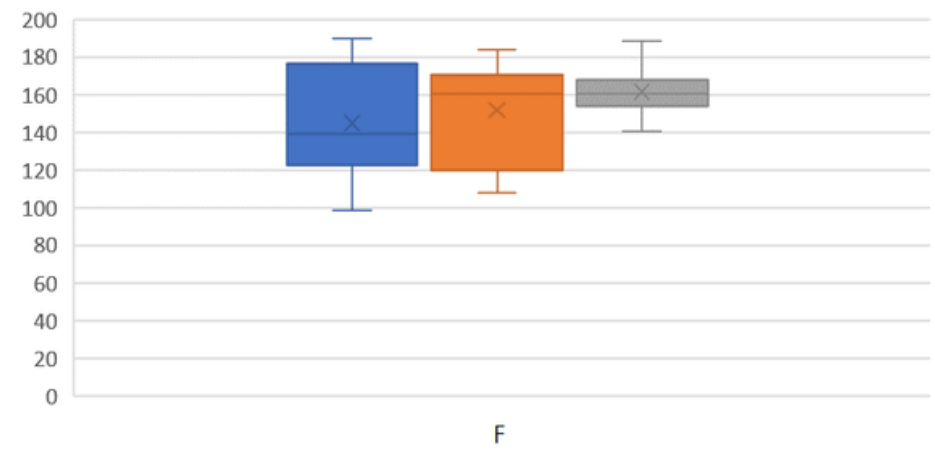

\section{Figure 3}

Box plot of IL-6s and IL-27s 


\section{ROC curve}

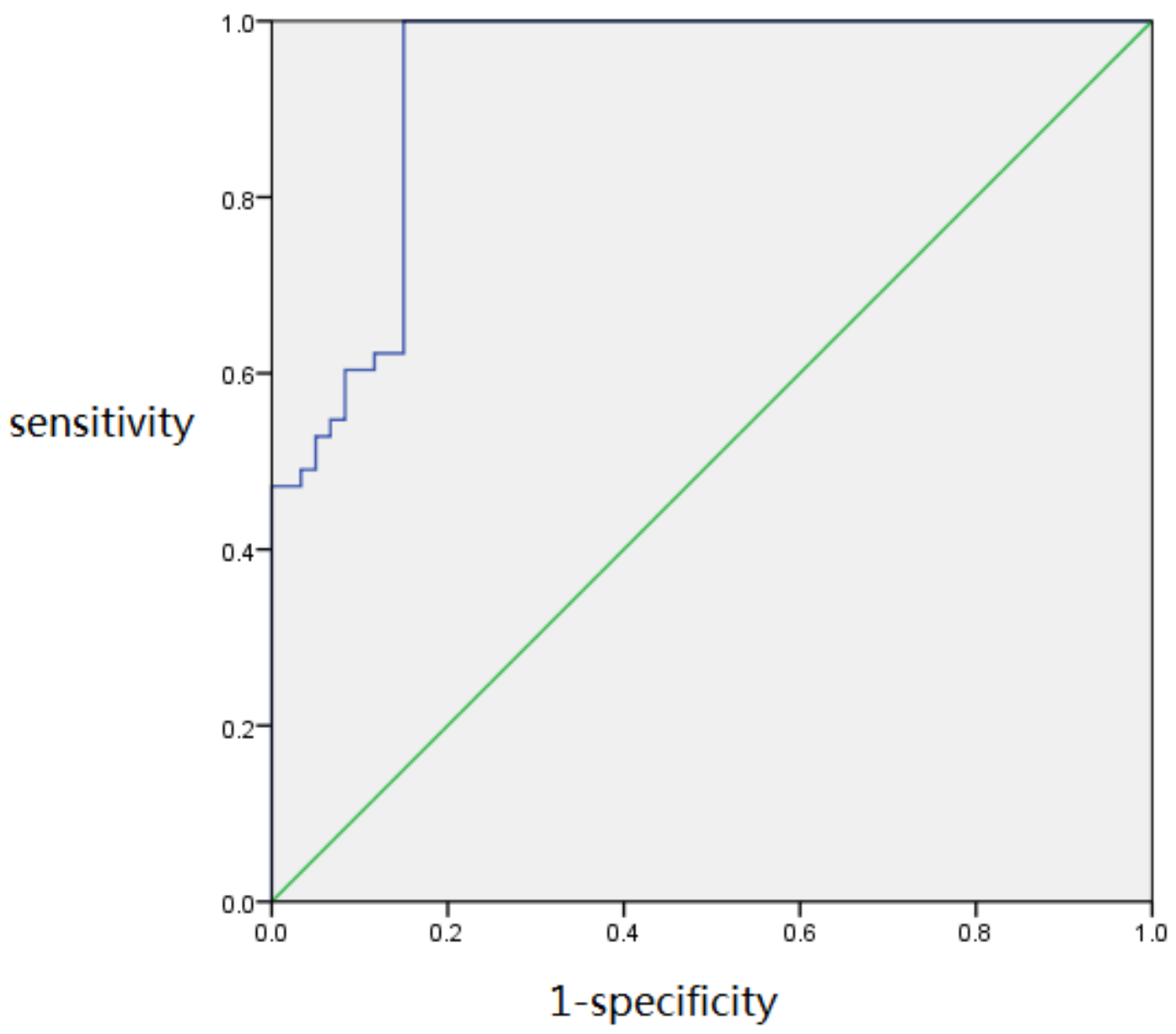

Figure 4

ROC curve analysis of IL-6s in mild case and severe cases of MPP
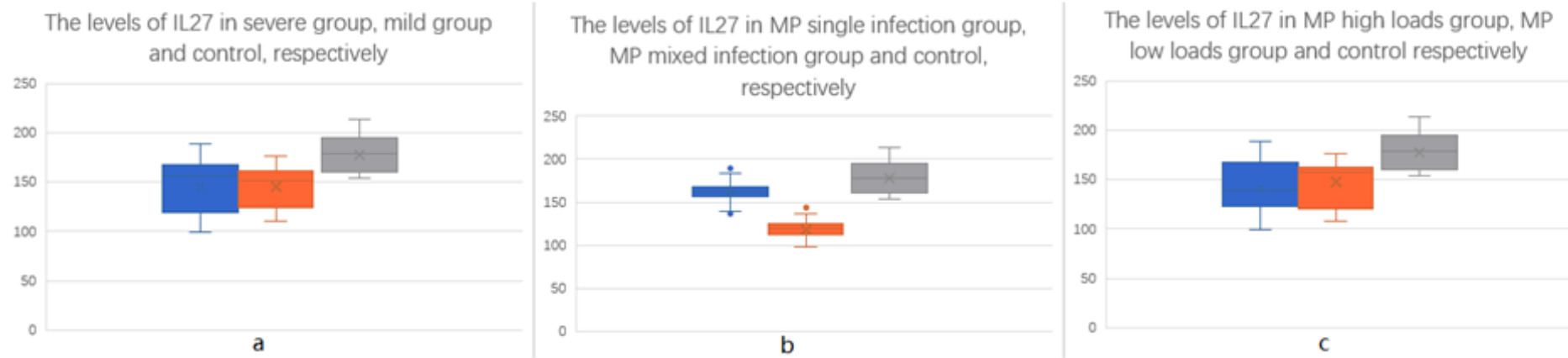

Figure 5

Box plot of IL-27s 